\section{Effects of vasoconstrictor on arterial blood pressure during minor oral surgical procedures}

\author{
Abubakar M. Kaura, ${ }^{1}$ Babatunde O. Bamgbose, ${ }^{1,2^{*}}$ SAB Ogunwande, ${ }^{3}$ \\ Olusola I. Amole, ${ }^{1}$ Junichi Asaumi, ${ }^{4}$ Thomas Owobu ${ }^{5}$
}

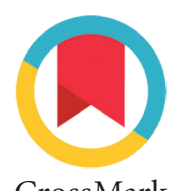

CrossMark

\title{
Abstract
}

Objective: A majority of minor oral surgical procedures require the use of local anaesthetics containing vasoconstrictors as part of the composition. These vasoconstrictors cause some hemodynamic changes either by direct action on the cardiac muscle or by stimulation of the autonomic innervations of the heart. Depending on the concentration of the vasoconstrictor, an increased heart rate, increased force of cardiac contraction, and ultimately increased blood pressure may ensue. The aim of this study was to assess the effect of vasoconstrictors contained in local anaesthetics on arterial blood pressure during minor oral surgical procedures.

Material and Methods: This was a one year prospective, randomized, comparative study involving the assessment of blood pressure and pulse rate by using an electronic digital blood pressure monitoring device amongst consenting patients who were referred for tooth extraction. The potential research participants were randomly allocated into either the study or the control group. The study group was treated using 2\% lignocaine with 1:80,000 epinephrine, while the control was treated using $2 \%$ plain lignocaine (without epinephrine). Normal or controlled-hypertensive patients between the ages of 18 to 55 years were enrolled into the study.

Results: A total of 100 subjects with a mean age of $34.66 \pm 10.3$ years participated in the study. There was no statistically significant difference in blood pressure and pulse rate between the patients in both the study group and control group.

Conclusion: Optimal use of adrenaline-containing local anaesthetic does not cause a statistically significant increase in blood pressure or pulse rate.
'Department of Oral and Maxillofacial Surgery, Faculty of Dentistry, Bayero University Kano and Aminu Kano Teaching Hospital Kano, Nigeria

${ }^{2}$ Department of Oral Diagnostic Sciences, Faculty of Dentistry, Bayero University Kano and Aminu Kano Teaching Hospital, Nigeria ${ }^{3}$ Department of Surgery, Bowen University Nigeria, Nigeria

${ }^{4}$ Department of Oral and Maxillofacial Radiology, Okayama University Graduate School of Medicine, Dentistry and Pharmaceutical Sciences, Japan ${ }^{5}$ Department of Dental Surgery, FMC Nguru, Yobe State, Nigeria

\section{*Corresponding to:}

Babatunde 0. Bamgbose,

Department of Oral and Maxillofacial Surgery, Faculty of Dentistry, Bayero University Kano and Aminu Kano Teaching Hospital Kano, Nigeria. Department of Oral Diagnostic Sciences, Faculty of Dentistry, Bayero University Kano and Aminu Kano Teaching Hospital, Nigeria drtundebamgbose@yahoo.com

Received: 11 July 2018

Revised: 1 August 2018

Accepted: 28 September 2018

Available Online 1 December 2018
Keywords: Vasoconstrictor, Blood pressure, Tooth extraction

Cite this Article: Kaura AM, Bamgbose B0, Ogunwande SAB, Amole 0l, Asaumi J, Owobu T. 2018. Effects of vasoconstrictor on arterial blood pressure during minor oral surgical procedures. Journal of Dentomaxillofacial Science 3(3): 136-143. D0I: 10.15562/jdmfs.v3i3.768

\section{Introduction}

Minor oral surgery is a broad term referring to outpatient surgical procedures of the oral cavity and jaws that can be performed safely and comfortably under general anaesthesia, local anaesthesia, or intravenous sedation..$^{1,2}$ It includes procedures that are well tolerated under local anaesthesia, such as surgical disimpaction of third molars, biopsies, frenectomies, reduction and immobilization of fractures. The currently used local anaesthetic agents consist vasoconstrictor as part of the composition to improve the biological properties of the solution. ${ }^{3}$ Of these vasoconstrictors, adrenaline is the most frequently used and its desirable properties include hemostasis, increased depth and duration of anaesthesia, decreased systemic absorption, decreased dose of anaesthetic agent required and, subsequently, reduced toxicity. ${ }^{4-7}$ These vasoconstrictors cause some hemodynamic changes which may be either by direct action on the cardiac muscle or by stimulation of the autonomic innervations of the heart. These effects may cause, depending on the concentration of the vasoconstrictor, an increased heart rate, increased force of cardiac contraction and, ultimately, increased blood pressure. ${ }^{8}$ However, the occurrence of most adverse reactions is due to inappropriate high dose and accidental injection into the vasculature. ${ }^{9,10}$

A handful of studies have been published on the hemodynamic changes following administration of vasoconstrictor-containing local anaesthetics. The results of these studies are mixed. However, only a few of these studies were from produced by Nigerian authors. Furthermore, the prevalence of hypertension in patients with oral diseases is increasing in Nigeria, ${ }^{11,12}$ which indirectly means that many hypertensive patients will require routine dental procedures under local anaesthesia. Therefore, there is need for more studies to determine the association between the vasoconstrictor (adrenaline) and blood pressure in the perioperative period in Nigerians.

The aim of this study was to assess the effect of a vasoconstrictor contained in local anaesthetics on arterial blood pressure during minor oral surgical procedures. The objective of the study was to determine the changes in Systolic Blood Pressure (SBP), Diastolic Blood Pressure (DBP), and pulse rate following administration of adrenalinecontaining local anaesthesia. 


\section{Material and Methods}

This was a prospective, randomized, comparative study involving the assessment of blood pressure and pulse rate changes that may occur following administration of local anaesthetic agent containing vasoconstrictor (epinephrine) during routine socket extraction of teeth at the Aminu Kano Teaching Hospital, Kano, over a period of one year. The potential research participants were randomly allocated into either the study or the control group. The study group was treated using $2 \%$ lignocaine with 1:80,000 epinephrine, while the control was treated using $2 \%$ lignocaine without epinephrine. The consenting study participants were in the age group of 18 to 55 years with normal or controlled blood pressure and were referred for tooth extraction at the Oral Surgery Clinic.

Ethical approval was obtained from the Research ethics review committee of Aminu Kano Teaching Hospital before the commencement of the study. The following categories of patients were excluded from the study; patients who met the inclusion criteria for minor oral surgical procedures but do not consent to participating in the study, patients with blood pressure $>140 / 90 \mathrm{mmHg}$, patients whose anxiety scores were high or very high, patients with systemic illness (e.g. thyrotoxicosis, uncontrolled diabetes mellitus), patients on medications like non-selective beta blockers, tricyclic antidepressants and phenothiazines, patients who were receiving treatment for psychiatric illness or suffering from any form of mental retardation.

Instruments of Measurement; Anxiety scale: numerical linear scale with zero being the lowest (no anxiety at all) and hundred being the highest score (the worst possible anxiety) was used to subjectively score the patients' anxiety level. Zero to twenty was translated as "low," twenty-one to forty as "medium," forty-one to sixty as "moderate," sixtyone to eighty as "high," and eighty one to hundred as "very high." Patients were asked to indicate a number on the linear scale that best described their anxiety for the dental extraction. Patients with high and very high anxiety scores were excluded from the study.

Blood pressure measuring device: a precalibrated non-invasive electronic digital blood pressure measuring device (EchoMax Plus ${ }^{\oplus}$ manufactured by Hubdic Co. Ltd Korea) was used for recording both the blood pressure and pulse rate of the patients.

Study protocol; the study and its procedures, including potential complications were explained to the participants, following which the informed consent documents were signed. The informed consent documents were translated and administered in the local language for non english speaking potential study participants. Potential study participants were then randomly allocated into either study or control group using simple random sampling (ballot method). The anxiety level was scored using a linear numerical scale with a score of zero to hundred. Participants with high and very high anxiety scores were excluded from the study. Baseline blood pressure and pulse rate of participants were measured 5 minutes before the administration of local anaesthesia. A maximum of two cartridges of the local anaesthetic (2\% lignocaine with 1:80,000 epinephrine) were given for each participant in the study group. A similar quantity of non-epinephrine containing $2 \%$ lignocaine was also administered to the control group. Aspirating dental syringe was used in both the study and control groups to avoid inadvertent intravascular injection. Participants requiring more than two cartridges for the procedure were dropped out of the study. The injection technique was dependent on the type of procedure to be performed and the anatomical site, whether maxillary or mandibular.

Blood pressure and pulse rate were checked and recorded 5 minutes after the local anaesthetic injection but before the onset of procedure; then another measurement was taken 2 minutes after commencing the procedure. Final recording of blood pressure and pulse rate was done 15 and 30 minutes after the procedure using a pre-calibrated digital blood pressure monitor. Blood pressure and pulse rate were measured twice at each instance and the average reading was recorded. Emergency medical kit was made available to manage emergencies that might arise. Patients were discharged after the procedure. Measurements of the blood pressure and pulse rates were done by the calibrated primary investigator.

Data analysis was performed using Statistical Package for Social Sciences Version 21.0 for Windows 7 (SPSS Inc. Chicago, IL, USA). A paired t-test was used for repeated measures in the same group while independent t-test was used to determine intergroup differences. Multivariate logistic regression was done to determine the influence of socio-demographic variables, anxiety level, number of cartridges used and injection technique on the outcome of the study. A confidence interval of $95 \%$ was used in this study and a p-value of less than 0.05 was considered significant.

\section{Results}

All the selected participants agreed and consented to participate in the study, giving a response rate of $100 \%$. None of the participants required more than two cartridges of local anaesthetics. Therefore, no patients were dropped out of the study. 
Table 1 Mean age of study participants

\begin{tabular}{lccc}
\hline Group & Mean Age (years) & Number & Std. Deviation p-value \\
\hline Study & 33.72 & 50 & 9.76 \\
Control & 35.6 & 50 & 10.9670 .367 \\
Total & 34.66 & 100 & 10.372 \\
\hline
\end{tabular}

Table 2 Socio-demographic characteristics of participants

\begin{tabular}{|c|c|c|c|}
\hline \multirow{2}{*}{$\begin{array}{l}\text { Socio-demographic } \\
\text { Variable }\end{array}$} & \multicolumn{2}{|c|}{ Group } & \multirow[b]{2}{*}{ p-value } \\
\hline & $\begin{array}{c}\text { Vasoconstrictor } \\
\text { n (\%) }\end{array}$ & $\begin{array}{c}\text { No Vasoconstrictor } \\
\text { n (\%) }\end{array}$ & \\
\hline \multicolumn{4}{|l|}{ Sex } \\
\hline Male & $20(40.0)$ & $19(38.0)$ & \multirow{3}{*}{0.838} \\
\hline Female & $30(60.0)$ & $31(62.0)$ & \\
\hline Total & $50(100)$ & $50(100)$ & \\
\hline \multicolumn{4}{|l|}{ Occupation } \\
\hline Students & $4(8.0)$ & $11(22.0)$ & \multirow{8}{*}{0.217} \\
\hline Dependents & $9(18.0)$ & $8(16.0)$ & \\
\hline Artisans & $5(10.0)$ & $1(2.0)$ & \\
\hline Traders & $10(20.0)$ & $11(22.0)$ & \\
\hline $\begin{array}{l}\text { Motorcyclists/ } \\
\text { Drivers }\end{array}$ & $2(4.0)$ & $1(2.0)$ & \\
\hline Civil servants & $19(38.0)$ & $17(34.0)$ & \\
\hline Professionals & $1(2.0)$ & $1(2.0)$ & \\
\hline Total & $50(100)$ & $50(100)$ & \\
\hline \multicolumn{4}{|l|}{ Ethnicity } \\
\hline Ibos & $2(4.0)$ & $1(2.0)$ & \multirow{6}{*}{0.157} \\
\hline Yorubas & $7(14.0)$ & $3(6.0)$ & \\
\hline Hausas & $36(72.0)$ & $40(80.0)$ & \\
\hline Fulanis & $1(2.0)$ & $5(10.0)$ & \\
\hline Others & $4(8.0)$ & $1(2.0)$ & \\
\hline Total & $50(100)$ & $50(100)$ & \\
\hline \multicolumn{4}{|l|}{ Nationality } \\
\hline Nigerians & $49(98.0)$ & $50(100)$ & \multirow{3}{*}{1.000} \\
\hline Non-Nigerian & $1(2.0)$ & $0(0.0)$ & \\
\hline Total & $50(100)$ & $50(100)$ & \\
\hline \multicolumn{4}{|c|}{ Highest educational level } \\
\hline Uneducated & $1(2.0)$ & $1(2.0)$ & \multirow{6}{*}{0.891} \\
\hline Primary & $6(12.0)$ & $3(6.0)$ & \\
\hline Secondary & $9(18.0)$ & $9(18.0)$ & \\
\hline Tertiary & $31(62.0)$ & $34(68.0)$ & \\
\hline Islamic/Arabic & $3(6.0)$ & $3(6.0)$ & \\
\hline Total & $50(100)$ & $50(100)$ & \\
\hline
\end{tabular}

Socio demographic data; a total of 100 subjects aged between 18 and 55 years participated in the study. The mean age of all the participants was $34.66 \pm 10.3$ years. There was no statistically significant difference in the mean age of respondents in the each group ( $\mathrm{p}=0.367$ ). Mean age in the study group (vasoconstrictor group) was $33.72 \pm 9.8$ years and the control group (without vasoconstrictor) was $35.60 \pm 10.9$ years table 1 . Study participants consisted of 39 (39\%) males and 61 (61\%) females. There were $20(40 \%)$ males and $30(60 \%)$ females in the study group, while the control group consisted of $19(38 \%)$ males and 31 (62\%) females table 2. Occupation of the participants was also fairly balanced as there were 19 (38\%) civil servants in the study group and 17 (34\%) in the control group that made up the largest percentage. This is followed by traders in which there were $10(20 \%)$ in the study group and $11(22 \%)$ in the control group. There were students $4(8 \%)$ in the study group and $11(22 \%)$ in the control group table 2. The major ethnic group amongst the participants was Hausa with 36 (72\%) and $40(80 \%)$ in the study and control groups, respectively. Other ethnic groups that participated in the study, in order of decreasing frequency, were Yoruba, Fulani and Ibo figure 1 and figure 2.

There was no statistically significant difference when the study and control groups were matched for gender, occupation, and ethnicity figure 1, figure 2 and table 2.

Pre-anaesthetic data; No statistically significant difference in the history of hypertension between the two groups $(\mathrm{p}=0.817)$ with a frequency of $12(24 \%)$ and $13(20 \%)$ in the study and control groups, respectively. The number of local anaesthetic cartridges used, and injection techniques were not statistically significant amongst the two groups with p-values of (0.155 and 0.305), respectively. However, there was statistically significant difference in terms of the level of anxiety between the two groups with a higher percentage of participants in the control group recording medium and moderate levels of anxiety ( $p$-value $=0.004$ ) table 3 .

Baseline hemodynamic data; the mean SBP before local anaesthesia in all the participants was $126.19 \pm 10.9 \mathrm{mmHg}$, with a mean of $126.80 \pm 11.4$ $\mathrm{mmHg}$ in the study group and $125.58 \pm 10.5$ in the control group. The mean DBP of all participants before local anaesthesia was $73.87 \pm 10.9 \mathrm{mmHg}$ with a mean of $74.32 \pm 10.9 \mathrm{mmHg}$ in the study group and $73.42 \pm 11.0 \mathrm{mmHg}$ in the control group. The mean pulse rate of all the participants before local anaesthesia was $88.11 \pm 16.5 \mathrm{bpm}$ with a mean value of $89.86 \pm 17.7 \mathrm{bpm}$ in the study group and $86.36 \pm 15.2 \mathrm{bpm}$ in the control group table 4 . Hemodynamic relationships of the study and control group; the hemodynamic relationship between the study and control group at different time intervals is detailed in tables 4-8. There was 
Table 3 Pre-anaesthetic evaluation of participants

\begin{tabular}{|c|c|c|c|}
\hline \multirow[b]{2}{*}{ Variable } & \multicolumn{2}{|c|}{ Group } & \multirow[b]{2}{*}{ p-value } \\
\hline & $\begin{array}{c}\text { Vasoconstrictor } \\
\mathbf{n}(\%)\end{array}$ & $\begin{array}{c}\text { No Vasoconstrictor } \\
\text { n (\%) }\end{array}$ & \\
\hline \multicolumn{4}{|c|}{ History of Hypertension } \\
\hline Yes & $12(24.0)$ & $13(26.0)$ & \multirow{3}{*}{0.817} \\
\hline No & $38(76.0)$ & $37(74.0)$ & \\
\hline Total & $50(100)$ & $50(100)$ & \\
\hline \multicolumn{4}{|l|}{ Anxiety level } \\
\hline Very low/low & $10(20.0)$ & $0(0.0)$ & \multirow{4}{*}{0.004} \\
\hline Medium & $16(32.0)$ & $21(42.0)$ & \\
\hline Moderate & $24(48.0)$ & $29(58.0)$ & \\
\hline Total & $50(100)$ & $50(100)$ & \\
\hline \multicolumn{4}{|c|}{ Number of Cartridges } \\
\hline 1 & $33(66.0)$ & $26(52.0)$ & \multirow{3}{*}{0.155} \\
\hline 2 & $17(34.0)$ & $24(48.0)$ & \\
\hline Total & $50(100)$ & $50(100)$ & \\
\hline \multicolumn{4}{|c|}{ Injection technique } \\
\hline Nerve block & $28(56.0)$ & $33(66.0)$ & \multirow{3}{*}{0.305} \\
\hline Infiltration & $22(44.0)$ & $17(34.0)$ & \\
\hline Total & $50(100)$ & $50(100)$ & \\
\hline
\end{tabular}

Table 4 Blood pressure and pulse rate 5 minutes before local anaesthetic injection

\begin{tabular}{llcccc}
\hline Parameter & Group & N & Mean & $\begin{array}{c}\text { Std. } \\
\text { Deviation }\end{array}$ & p-value \\
\hline Systolic Blood & Vasoconstrictor & 50 & 126.80 & 11.452 & 0.580 \\
Pressure & No Vasoconstrictor & 50 & 125.58 & 10.510 & \\
Diastolic Blood & Vasoconstrictor & 50 & 74.32 & 10.927 & 0.683 \\
Pressure & No Vasoconstrictor & 50 & 73.42 & 11.073 & \\
Pulse Rate & Vasoconstrictor & 50 & 89.86 & 17.757 & 0.293 \\
& No Vasoconstrictor & 50 & 86.36 & 15.275 & \\
\hline
\end{tabular}

Table 5 Blood pressure and pulse rate 5 minutes after the local anaesthetic injection

\begin{tabular}{llcccc}
\hline Parameter & Group & N & Mean & Std. Deviation & p-value \\
\hline Systolic Blood & Vasoconstrictor & 50 & 125.36 & 13.953 & 0.408 \\
Pressure & No & 50 & 122.98 & 14.667 & \\
& Vasoconstrictor & & & & \\
Diastolic Blood & Vasoconstrictor & 50 & 71.36 & 11.602 & 0.502 \\
Pressure & No & 50 & 69.90 & 9.988 & \\
& Vasoconstrictor & & & & \\
Pulse Rate & Vasoconstrictor & 50 & 92.44 & 18.495 & 0.248 \\
& No & 50 & 88.52 & 15.096 & \\
& Vasoconstrictor & & & & \\
\hline
\end{tabular}

\section{Percentages of different tribes}

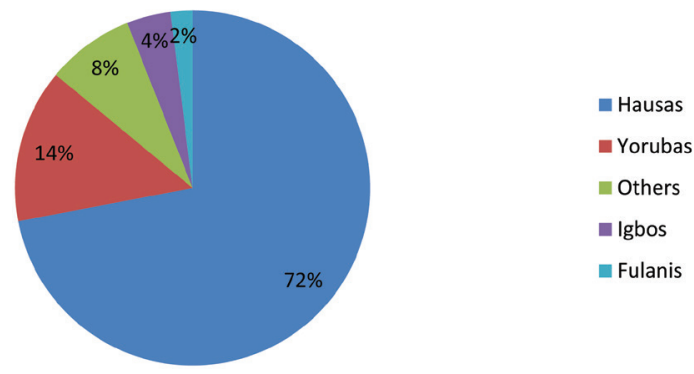

Figure 1 A pie chart showing the ethnic distribution in the study group

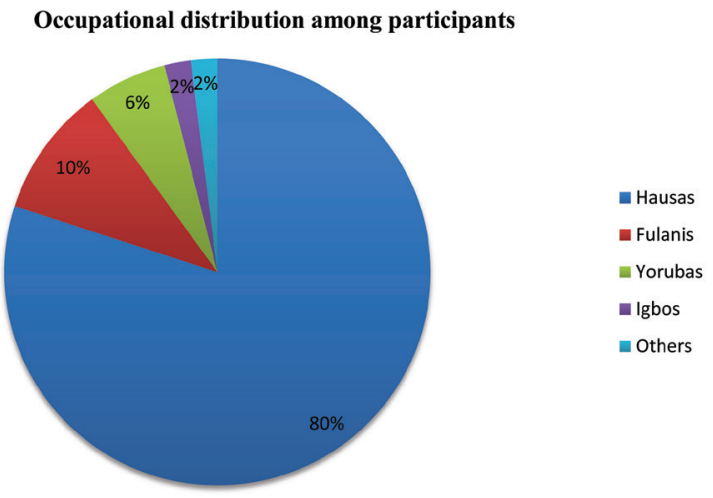

Figure 2 A pie chart showing the ethnic distribution among the control group

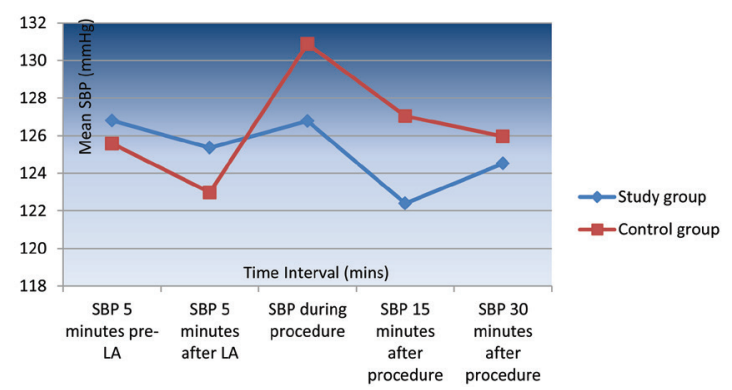

Figure 3 Comparison of mean SBP between study and control groups

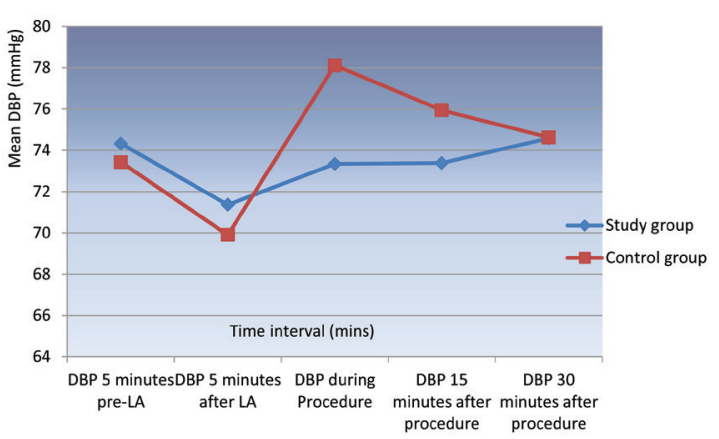

Figure 4 Comparison of mean diastolic blood pressure between study and control groups 
Table 6 Blood pressure and pulse rate during the procedure

\begin{tabular}{llcccc}
\hline Parameter & Group & N & Mean & $\begin{array}{c}\text { Std. } \\
\text { Deviation }\end{array}$ & p-value \\
\hline Systolic Blood & Vasoconstrictor & 50 & 126.78 & 15.700 & 0.195 \\
Pressure & No Vasoconstrictor & 50 & 130.88 & 15.733 & \\
Diastolic Blood & Vasoconstrictor & 50 & 73.34 & 12.328 & 0.060 \\
Pressure & No Vasoconstrictor & 50 & 78.12 & 12.758 & \\
Pulse Rate & Vasoconstrictor & 50 & 91.26 & 19.624 & 0.134 \\
& No Vasoconstrictor & 50 & 85.86 & 15.869 & \\
\hline
\end{tabular}

Table 7 Blood pressure and pulse rate 15 minutes after the procedure

\begin{tabular}{llcccc}
\hline Parameter & Group & N & Mean & $\begin{array}{c}\text { Std. } \\
\text { Deviation }\end{array}$ & p-value \\
\hline Systolic Blood & Vasoconstrictor & 50 & 122.40 & 14.737 & 0.098 \\
Pressure & No Vasoconstrictor & 50 & 127.04 & 12.965 & \\
Diastolic Blood & Vasoconstrictor & 50 & 73.38 & 12.763 & \multirow{2}{*}{0.303} \\
Pressure & No Vasoconstrictor & 50 & 75.94 & 11.965 & \\
Pulse Rate & Vasoconstrictor & 50 & 85.06 & 15.990 & \multirow{2}{*}{0.377} \\
& No Vasoconstrictor & 50 & 82.34 & 14.649 & \\
\hline
\end{tabular}

\section{Table 8 Blood pressure and pulse rate $\mathbf{3 0}$ minutes after the} procedure

\begin{tabular}{llcccc}
\hline Parameter & Group & N & Mean & $\begin{array}{c}\text { Std. } \\
\text { Deviation }\end{array}$ & p-value \\
\hline Systolic Blood & Vasoconstrictor & 50 & 124.52 & 12.453 & 0.545 \\
Pressure & No Vasoconstrictor & 50 & 125.96 & 11.234 & \\
Diastolic Blood & Vasoconstrictor & 50 & 74.58 & 10.218 & 0.985 \\
Pressure & No Vasoconstrictor & 50 & 74.62 & 10.616 & \\
Pulse Rate & Vasoconstrictor & 50 & 85.58 & 15.786 & 0.800 \\
& No Vasoconstrictor & 50 & 84.82 & 14.095 & \\
\hline
\end{tabular}

\section{Table 9 Multivariate Logistic Regression Model}

\begin{tabular}{|c|c|c|c|c|c|}
\hline \multirow[b]{2}{*}{ Parameter } & \multirow[b]{2}{*}{ B } & \multirow[b]{2}{*}{ p-value } & \multirow{2}{*}{$\begin{array}{c}\text { Adjusted } \\
\text { Odds Ratio }\end{array}$} & \multicolumn{2}{|c|}{$\begin{array}{c}95 \% \mathrm{Cl} \text { of Odds } \\
\text { Ratio }\end{array}$} \\
\hline & & & & Lower & Upper \\
\hline Sex & 0.647 & 0.340 & 1.910 & 0.51 & 7.21 \\
\hline Occupation & 1.666 & 0.327 & 5.289 & 0.19 & 147.74 \\
\hline Ethnicity & 0.407 & 0.838 & 1.503 & 0.03 & 73.74 \\
\hline $\begin{array}{l}\text { Highest Educational } \\
\text { Level }\end{array}$ & 0.113 & 0.959 & 1.119 & 0.02 & 81.14 \\
\hline Anxiety Level & -0.488 & 0.444 & 0.614 & 0.18 & 2.14 \\
\hline No of Cartridge & -0.691 & 0.198 & 0.501 & 0.17 & 1.44 \\
\hline Injection Technique & 0.260 & 0.636 & 1.297 & 0.44 & 3.80 \\
\hline Constant & -1.239 & 1.000 & 0.290 & & \\
\hline
\end{tabular}

$\mathrm{B}=$ Coefficient of Regression, $\mathrm{CI}=$ Confidence Interval

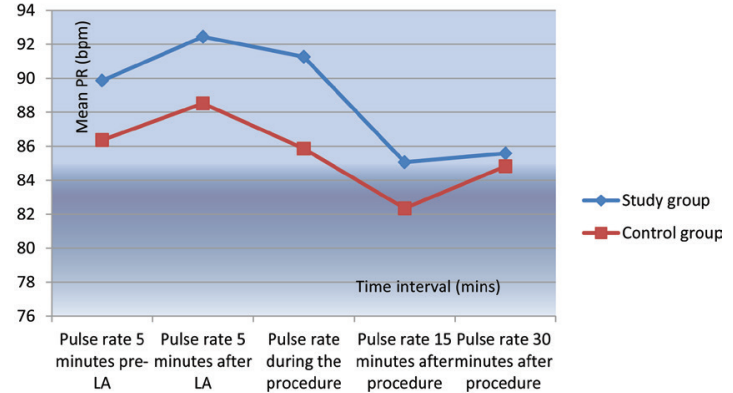

Figure 5 Comparison of pulse rate between study and control groups

no statistically significant difference between the two groups at all intervals. However, the highest variation found in the blood pressure was during the procedure in which there was increase in both the mean systolic and DBP in the control group table 6.

The different pattern in mean SBP in the two groups is depicted in figure 1. There was a slight decrease in the mean SBP from the baseline value of $126.80 \mathrm{mmHg}$ to $125.36 \mathrm{mmHg}$ in the study group, and from $125.58 \mathrm{mmHg}$ to $122.98 \mathrm{mmHg}$ in the control group, 5 minutes after local anaesthetic injection. After this decrease, there was an increase in the mean SBP to about $126.78 \mathrm{mmHg}$ and $130.88 \mathrm{mmHg}$ in the study and control groups respectively (the rise was sharper in the control group than in the study group). The mean SBP dropped down to near pre-local anaesthetic level in both groups after about 30 minutes. The mean DBP also followed the same pattern of fluctuation. It decreased from the baseline value of 74.32 $\mathrm{mmHg}$ to $71.36 \mathrm{mmHg}$ in the study group, and from $73.42 \mathrm{mmHg}$ to $69.90 \mathrm{mmHg}$ in the control group. There was a remarkable upsurge from the post-anaesthetic value to about $78.12 \mathrm{mmHg}$ in the control group. The values in both groups also came to near pre-anaesthetic reading in about 30 minutes figure 2 . The pulse rate also showed a constant pattern of higher value in the study group as compared to the control group at all intervals. Furthermore, there was clear depiction of physiological response to decrease in pulse rate by increase in blood pressure figure 3. Upon analysis using multivariate logistic regression model, none of the parameters was found to be an independent predictor of difference in the BP and PR between the study and control groups table 9 .

\section{Discussion}

Majority of minor oral surgical procedures require the use of local anaesthetics. ${ }^{5,6}$ The composition of local anaesthetic agents consist of a vasoconstrictor 
to improve the biological properties of the solution which may be either adrenergic agonist or felypressin. ${ }^{3}$ Adrenaline is the most frequently used vasoconstrictor in daily dental practice to achieve the desired anaesthetic properties, including hemostasis, increased depth and duration of anaesthesia, decreased systemic absorption, decreased dose of anaesthetic agent required and subsequently reduced toxicity. ${ }^{4-7}$ Vasoconstrictors can cause some hemodynamic changes either by direct action on the cardiac muscle or by stimulation of the autonomic innervations of the heart. These may cause, depending on the concentration of the vasoconstrictor, an increased heart rate, increased force of cardiac contraction and ultimately increased blood pressure. ${ }^{8}$ However, the occurrence of most adverse reactions is usually due to inappropriate high dose injection and accidental injection into the vasculature. ${ }^{9,10}$ Many authors concluded that adrenaline has a safety range but its threshold in cardiovascular patients is not yet clear. ${ }^{13,14}$ However, controversies still exist as regards the concentration of adrenaline in local anaesthetic solutions and its mode of usage. ${ }^{16}$ Furthermore, the fact that adrenaline is a vasopressor agent can explain why it is potentially implicated as the causative agent in certain clinical situations of hypertension encountered in the dental office. ${ }^{15,16}$ Hypertensive patients represent an important risk group among patients visiting the dental clinic as many of them give a history of high blood pressure. ${ }^{15}$ This is more so in an underdeveloped economy, like Nigeria, where routine medical check-ups are not done and many individuals do not know whether they are hypertensive or not. It, therefore, becomes important to check blood pressure of dental patients routinely.

The age of the patients that participated in this study differed from those that participated in the study conducted by Ogunlewe et al. $(50.1 \pm 11.7)$ but similar to Mohammed et al. ${ }^{18}(35.45 \pm 7.99)$ study included only hypertensive patients with the likelihood of having more hypertensive patients among the middle age and elderly group. ${ }^{17}$

The gender of the participants in our study had some similarities and differences to the previously conducted studies. In our study there was a slightly higher female to male ratio of about 1.5:1. ${ }^{17}$ A higher female to male ratio was reported by Gungormuset et al. ${ }^{4}$ with a female to male ratio of about 2:1. However, different findings were reported by Mohammed et al..$^{18}$ and Marcelo et al. ${ }^{19}$ with a female to male ratio of about 1:1. The higher ratio female to male in our study may be because, traditionally, females have low pain threshold compared to males and they tend to seek medical attention more than their male counterparts.
Our study showed no statistically significant changes in the SBP, DBP and PR following administration of $2 \%$ lignocaine with 1: 80,000 epinephrine. This is similar to reports by Gungormus et al., ${ }^{4}$ Chaudary et al..$^{20}$ Ogunlewe et al. ${ }^{17}$ and de Morais et al. ${ }^{21}$ On the other hand, Mohammad et al. ${ }^{18}$ reported a statistically significant difference in all the three hemodynamic parameters (SBP, DBP \& PR) after administration of one cartridge of $2 \%$ lignocaine with 1:80,000 adrenaline. Study used different anaesthetic agents for study and control groups (2\% lignocaine $+1: 80,000$ adrenaline and $3 \%$ mepivacaine respectively). This may be a source of bias and unequal comparison in the groups, especially because confounders, like anxiety, were not recorded. ${ }^{18}$

A slight decrease in mean SBP was observed in the present study after local anaesthetic administration. The decrease was more in the control group than in the study group. Similar finding was observed by Chaudary et al. ${ }^{17}$ They reported a decrease in SBP after a local anaesthetic agent containing adrenaline was injected in patients with stage 2 hypertension undergoing tooth extraction. This is, however, is contrary to the observations of Akinmoladun et al., ${ }^{22}$ Nedal et al., ${ }^{23}$ and Ogunlewe et al. ${ }^{24}$ The latter reported slight increase in the mean SBP after administration of local anaesthetic agent containing adrenaline administration. Nedal et al. ${ }^{23}$ conducted their study using three local anaesthetic agents with different concentrations of adrenaline (2\% lignocaine with 1:80.000 epinephrine, 4\% articaine with 1: 100.000 epinephrine and $4 \%$ articaine with 1: 200.000 epinephrine). Hemodynamic parameters were measured 3 minutes before local aneasthetic, 3 minutes after LA, and 3 minutes after extraction. They reported a significant increase in SBP 3 minutes after local anaesthetic administration in the group treated using $2 \%$ lignocaine with 1:80.000 epinephrine. The decrease in SBP after local anaesthetic administration observed in our study could be explained in two ways. First, given that lignocaine is a potent vasodilator; its vasodilator effect may take precedence over the vasoconstrictor effect of the adrenaline. This may further explain why the reduction is more pronounced in the group treated with plain lignocaine. Secondly, the anxiety associated with an intra-oral injection resulting in slightly elevated baseline blood pressure is eliminated as soon as the injection is given which, consequently, may reduce the blood pressure. Lastly, the pain that might have been the indication for the tooth extraction, which could also trigger endogenous catecholamine release, was abated as soon as the local anaesthetic agent takes effect. 
There was a pronounced increase in SBP in those patients whose minor surgical procedure was done using plain lignocaine. Plain lignocaine is considered to possess less depth and duration of action when compared to adrenaline containing ligno caine $\mathrm{HCl}$. This may result in poor pain control with pain-induced release of endogenous catechol-amines which may lead to elevated blood pressure. ${ }^{27}$ On the other hand, the vasoconstrictor group (study group) did not exhibit remarkable change in blood pressure possibly due to profound anaesthesia which eliminates the physiological response of releasing the endogenous catecholamines to pain and stress. Our study observed that anxiety and socio demographic variables cannot predict the change in blood pressure or pulse rate when we considered the use of vasoconstrictor or lack of it in the local anaesthetic agent. The pulse rate slightly increased after the local anaestheti administration, it then continued to decrease during the procedure until it reached a value below the preanaesthetic level 15 minutes after the procedure. It then rose to almost pre-anaesthetic value 30 minutes after the procedure. It is generally accepted that the pulse rate increases when there is a decrease in blood pressure and vice versa. This study observed a similar pattern, as there was an increase in pulse rate 5 minutes after the local anaesthetic administration which corresponded to the time when both the SBP and DBP had decreased and there was decrease in pulse rate during the procedure which also corresponded to when both the SBP and DBP had increased.

\section{Conclusion}

The use of adrenaline-containing local anaesthetics is more favourable to the hemodynamic variables, both in controlled hypertensive and normotensive patients, than the plain local anaesthetics due to their lack of adverse effects on blood pressure and pulse rate as well as more effective pain control during the procedure.

\section{Acknolegment}

The authors express my gratitude to Prof. Mrs J.O Taiwo, Dr Rayyan Garba, and Dr Sani Balarabe for their immense support towards the successful completion of this study.

\section{Conflict of Interest Statement}

The authors declared no conflicts of interest in this study.

\section{Human Rights Statement}

Ethical approval was obtained from the research ethical review committee of Aminu Kano Teaching Hospital before the commencement of the study. The procedures in the study were in accordance with the ethical standards of responsible conduct of research and the Helsinki Declaration of 1975, as revised in 2008.

\section{References}

1. Clayton L, Thomas. Taber's cyclopedic medical dictionary. 18th ed. Philadelphia: F.A Davis; 1997. p. 1868.

2. Gregory PH. Protocol for the management of oral surgery patients on warfarin utilizing a Point-of-Care In-Office international normalized ratio monitoring device. Open J Stomatol 2013;3: 255-267.

3. Brown G. The influence of adrenaline, noradrenaline vasoconstrictors on the efficacy of lidocaine. J Oral Ther Pharmacol 1968;4: 398-405.

4. Gungormus M, Buyukkurt MC. The evaluation of changes in blood pressure and pulse rate of hypertensive patients during Tooth Extraction. Acta Med Austriaca 2003;30: 127-129.

5. Haas DA. An update on local anaesthetics in dentistry. J Can Assoc 2002;68: 546-551.

6. Sotunmbi PT, Arotiba JT. A manual of anaesthesia for dental and maxillofacial surgery. 1st ed. Nigeria: Ambod Publishers; 2005. p. 72-102.

7. Brand HS. Cardiovascular responses in patient and dentist during dental treatment. Int Dent J 1999;49: 60-66.

8. Maria TFC, Ana Cristina PL, Fabio SB, et al. Effect of local anesthetics with and without vasconstrictor agent in patients with ventricular arrhythmias. Arq Bras Cardiol 2008;91: 128-133.

9. Yagiela JA, Duffin SR, Hunt IM. Drug interactions and vasoconstritors used local solutions. Oral Surg Oral Med Oral Pathol 1985;59: 565-571.

10. Perusse R, Goulet JP, Turcotte JY. Contraindications to vasoconstrictor in dentistry: Part I. Cardiovascular diseases. Oral Surg Oral Med Oral Pathol 1992;74: 679-686.

11. Adediran OS, Okpara IC, Adeniyi OS, et al. Hypertension prevalence in an urban and rural area of Nigeria. J Med Med Sci 2013;4: 149-154.

12. Akpata ES. Oral health in Nigeria. Int Dent J 2004;54: 361-366.

13. Niwa H, Sugimura M, Satoh Y, Tanimoto A. Cardiovascular response to epinephrine-containing local anesthesia in patients with cardiovascular disease. Oral Surg Oral Med Oral Pathol Oral Radiol Endod 2001;92: 610-616.

14. Cioffi GA, Chernow B, Glahn RP, Terezhalmy GT, Lake CR. The hemodynamic and plasma catecholamine responses to routine restorative dental care. J Am Dent Assoc 1985;111: 67-70.

15. Muzkeya BC, Glick M. The hypertensive dental patient. J Am Dent Assoc 1997;128: 1109-1120.

16. Agency for Health Research and Quality (AHQR); Cardiovascular Effects of epinephrine in hypertensive dental patients; Number 48 AHQR Publication 02 -E005, March 2002.

17. Ogunlewe MO, James O, Ajuluchukwu JN, et al. Evaluation of hemodynamic changes in hypertensive patients during tooth extraction under local anesthesia. West Indian Med J 2011;60: 91-95.

18. Mohammad AG, Javad Y, Arezo GZ, et al. Comparison of heart rate and blood pressure after administration of anesthetic agent with and without vasoconstrictor. Int J Curr Res Acad Rev 2014;2: 153-158. 
19. Marcelo C, Bortoluzzi, Rafael M, et al. Glucose levels and hemodynamic changes in patients submitted to routine dental treatment with and without local anesthesia. Clin 2010;65: 975-978.

20. Chaudary S, Iqbal HA, Izhar F et al. Effect on blood pressure and pulse rate after administration of an epinephrine containing dental local anesthetic in hypertensive patients. J Pak Med Assoc 2011;61: 1088-1099.

21. de-Morais HH, de-Santana ST, da-Costa FA, et al. Hemodynamic changes comparing $2 \%$ lidocaine and $4 \%$ articaine with epinephrine 1: 100,000 in lower third molar surgery. J Craniofac Surg 2012;23: 1204-1211.

22. Akinmoladun VN, Okoje OM, Akinosun AO, et al. Evaluation of haemodynamic and metabolic effects of local anaesthetic agent in routine dental extractions. J Maxillofac Oral Surg 2013;12: 424-428.
23. Nedal AM, Fatimah A, Kawther A, et al. hemodynamic changes following injection of local anesthetics with different concentrations of epinephrine during simple tooth extraction: a prospective randomized clinical trial. J Clin Exp Dent 2015;7: e471-e476.

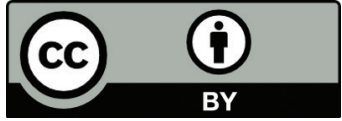

This work is licensed under a Creative Commons Attribution 